\title{
Cotrimoxazole enhances the in vitro susceptibility of Coccidioides posadasii to antifungals
}

\author{
Rossana de Aguiar Cordeiro ${ }^{1,2} /+$, Delia Jessica Astete-Medrano², \\ Francisca Jakelyne de Farias Marques ${ }^{1}$, Heuziwanne Tavares Leite Andrade ${ }^{1}$, \\ Lauro Vieira Perdigão Neto ${ }^{1}$, Juliane Lira Tavares ${ }^{3}$, Rita Amanda Chaves de Lima', \\ Kharla Kharolyni Nobre Rabelo Patoilo', Andre Jalles Monteiro4, \\ Raimunda Sâmia Nogueira Brilhante ${ }^{1,2}$, Marcos Fábio Gadelha Rocha ${ }^{1,5}$, \\ Zoilo Pires de Camargo ${ }^{6}$, José Júlio Costa Sidrim ${ }^{1,2}$
}

\footnotetext{
${ }^{1}$ Centro Especializado em Micologia Médica ${ }^{2}$ Programa de Pós-Graduação em Ciências Médicas ${ }^{4}$ Departamento de Estatística, Universidade Federal do Ceará, Fortaleza, CE, Brasil ${ }^{3}$ Departamento de Química ${ }^{5}$ Programa de Pós-Graduação em Ciência Veterinária, Universidade Estadual do Ceará, Fortaleza, CE, Brasil ${ }^{6}$ Departamento de Microbiologia, Imunologia e Parasitologia, Universidade Federal de São Paulo, São Paulo, SP, Brasil
}

The aim of the present study was to evaluate the effect of cotrimoxazole on the in vitro susceptibility of Coccidioides posadasii strains to antifungals. A total of 18 strains of C. posadasii isolated in Brazil were evaluated in this study. The assays were performed in accordance with the Clinical and Laboratory Standards Institute guidelines and the combinations were tested using the checkerboard method. The minimum inhibitory concentrations were reduced by 11, 2.4, 4.3 and 3.5 times for amphotericin B, itraconazole, fluconazole and voriconazole, respectively. Moreover, it was seen that cotrimoxazole itself inhibited C. posadasii strains in vitro. The impairment of folic acid synthesis may be a potential antifungal target for C. posadasii.

Key words: Coccidioides posadasii - cotrimoxazole - antifungals - susceptibility - antimicrobial synergism

In the recent years, several studies have shown the antifungal effect of "non-antifungal drugs" against true pathogenic species (Afeltra \& Verweij 2003, Chapman et al. 2008, Cordeiro et al. 2009). The search for new antifungals is warranted because of the limited number of therapeutic drugs available for treating these infections. In addition, many researchers have been trying to improve the results obtained with antifungal monotherapy. Although data from controlled clinical trials are scarce, many recent reports have claimed beneficial effects of antifungal combinations for the treatment of severe fungal infections (Johnson \& Perfect 2010, Matsuda et al. 2010).

Coccidioidomycosis is a deep fungal infection caused by the soil-dwelling ascomycetes Coccidioides spp. The disease has a broad clinical spectrum, ranging from a mild respiratory syndrome to progressive pneumonia and meningitis (Galgiani et al. 2005). Although Coccidioides spp rarely display antifungal resistance in vitro (Kriesel et al. 2008), specialists recognise that coccidioidomycosis is one of the most refractory fungal infections (Stevens et al. 2007). Therefore, several in vitro and in vivo studies have been performed in an attempt

Financial support: CNPq (620160/2008-0), CAPES (2103/2009)

+ Corresponding author: rossanacordeiro@ufc.br

Received 13 October 2010

Accepted 23 August 2011 to identify new potential therapeutic drugs and antimicrobial combinations that can be used against $\mathrm{Coc}$ cidioides spp (Shubitz et al. 2006, González et al. 2007, Cordeiro et al. 2009).

In this study, we investigated the effect of cotrimoxazole - a wide-spectrum antimicrobial formed by sulfamethoxazole (SMX) plus trimethoprim (TMP) - on the in vitro susceptibility of Coccidioides posadasii to amphotericin B (AMB), currently the most important drug used to treat life-threatening forms of coccidioidomycosis.

A total of 18 strains of $C$. posadasii isolated in the state of Ceará (Northeast Brazil) from clinical $(n=15)$ and environmental $(n=3)$ sources were evaluated in this study. The strains of $C$. posadasii were obtained from storage in $0.9 \%$ saline at $4^{\circ} \mathrm{C}$, subcultured on Sabouraud glucose agar (Difco, Detroit, USA) and incubated at $25^{\circ} \mathrm{C}$ for 10 days. Prior to antimicrobial testing, the viability and purity of each isolate were evaluated by microscopic examinations and polymerase chain reaction experiments described elsewhere (Cordeiro et al. 2010). All procedures were performed within a class II biological safety cabinet in a biosafety level 3 laboratory.

Stock solutions of AMB (Sigma Chemical Co, USA), itraconazole (ITR) (Janssen Pharmaceutica, Belgium) and voriconazole (VRZ) (Pfizer Pharmaceuticals, USA) were prepared in dimethyl sulfoxide (Sigma Chemical Co, USA). Fluconazole (FLC) (Pfizer Pharmaceuticals, USA) was prepared in distilled water according to Clinical and Laboratory Standards Institute (CLSI 2008) SMX plus TMP (Hipolabor Ind Farmacêutica Ltda, Brazil) and SMX/TMP/AMB combinations were prepared in Roswell Park Memorial Institute (RPMI) 1640 me- 
dium with L-glutamine and without sodium bicarbonate (Sigma Chemical Co, St. Louis, MO, USA) and buffered with 0.165 M MOPS (Sigma Chemical Co, USA). Serial two-fold dilutions of each antimicrobial combination were performed in RPMI 1640 medium.

Inoculum preparation was carried out as described by Cordeiro et al. (2009). Sterile normal saline was added to each agar slant and the cultures were gently scraped with cotton swabs. The suspension was transferred to a sterile tube and allowed to settle for $5 \mathrm{~min}$ and then the transmittance of the upper homogeneous supernatant was read at $530 \mathrm{~nm}$ and adjusted to $95 \%$ transmittance. The suspension containing arthroconidia and hyphae was diluted 1:10 with RPMI 1640 medium containing Lglutamine and without sodium bicarbonate and buffered to $\mathrm{pH} 7.0$ with $0.165 \mathrm{M}$ MOPS to obtain an inoculum of approximately $1 \times 10^{3}-5 \times 10^{3} \mathrm{CFU} / \mathrm{mL}$.

Antifungal susceptibility assays were performed by the broth macrodilution method (M38-A2) according to CLSI (2008) standards guidelines. First, the strains were tested against each antimicrobial alone to deter- mine the minimum inhibitory concentrations (MICs). The drug concentration ranges tested were as follows: AMB, 0.0625-1.0 $\mu \mathrm{g} / \mathrm{mL}$; ITR, 0.0625-1.0 $\mu \mathrm{g} / \mathrm{mL}$; VRZ, 0.031-0.5 $\mu \mathrm{g} / \mathrm{mL}$; FLC, 0.78-12.5 $\mu \mathrm{g} / \mathrm{mL}$; SMX-TMP, $250 / 50-4,000 / 800 \mu \mathrm{g} / \mathrm{mL}$. The procedures were repeated at least twice and each fungal strain was tested in duplicate. MICs for AMB and azoles were defined as the lowest concentration of the drug at which there was no visible fungal growth (CLSI 2008). We defined the MIC of SMX/TMP as the lowest drug concentration that caused $80 \%$ inhibition of visible fungal growth. After MIC definition for each drug, the strains were tested against the combination AMB/SMX/TMP. The following concentrations were tested: AMB, 0.007-0.116 $\mu \mathrm{g} / \mathrm{mL}$; SMX/ TMP, $125 / 25-2,000 / 400 \mu \mathrm{g} / \mathrm{mL}$. The MIC of each drug in combination was defined as the lowest concentration that caused $80 \%$ inhibition of visible fungal growth.

The non-parametric Wilcoxon Signed Rank Test was used for analysis of the antimicrobial combinations. The results were expressed as the mean and a $\mathrm{p}$ value of $<$ 0.05 was considered significant.

TABLE I

Minimum inhibitory concentration (MIC) of sulfamethoxazole plus trimethoprim (SMX/TMP) and antifungals against clinical and environmental Coccidioides posadasii strains

\begin{tabular}{|c|c|c|c|c|c|}
\hline \multirow[b]{2}{*}{ Strain } & \multicolumn{5}{|c|}{$\mathrm{MIC}(\mu \mathrm{g} / \mathrm{mL})$} \\
\hline & AMB & ITR & FLC & VRZ & SMX/TMP \\
\hline \multicolumn{6}{|l|}{ Clinical source } \\
\hline $01-6-085$ & 0.125 & 0.125 & 3.125 & 0.125 & $1,000 / 200$ \\
\hline $01-6-087$ & 0.125 & 0.125 & 3.125 & 0.125 & $2,000 / 400$ \\
\hline $01-6-088$ & 0.125 & 0.125 & 3.125 & 0.125 & $2,000 / 400$ \\
\hline 01-6-089 & 0.125 & 0.125 & 3.125 & 0.125 & $2,000 / 400$ \\
\hline $01-6-101$ & 0.0625 & 0.125 & 6.25 & 0.125 & $2,000 / 400$ \\
\hline 01-6-102 & 0.125 & 0.125 & 6.25 & 0.125 & $2,000 / 400$ \\
\hline $01-6-103$ & 0.125 & 0.5 & 6.25 & 0.125 & $1,000 / 200$ \\
\hline $05-02-063$ & 0.125 & 0.125 & 6.25 & 0.25 & $2,000 / 400$ \\
\hline $05-02-064$ & 0.125 & 0.25 & 6.25 & 0.25 & $2,000 / 400$ \\
\hline $05-02-065$ & 0.125 & 0.125 & 6.25 & 0.125 & $2,000 / 400$ \\
\hline $05-02-066$ & 0.0625 & 0.25 & 6.25 & 0.25 & $2,000 / 400$ \\
\hline $05-02-067$ & 0.125 & 0.125 & 3.125 & 0.125 & $2,000 / 400$ \\
\hline $05-02-068$ & 0.125 & 0.125 & 6.25 & 0.125 & $1,000 / 200$ \\
\hline $05-02-069$ & 0.125 & 0.125 & 6.25 & 0.125 & $1,000 / 200$ \\
\hline $05-02-070$ & 0.0625 & 0.125 & 6.25 & 0.125 & $2,000 / 400$ \\
\hline \multicolumn{6}{|c|}{ Environmental source } \\
\hline 01-6-090 & 0.125 & 0.25 & 6.25 & 0.125 & $2,000 / 400$ \\
\hline 01-6-091 & 0.125 & 0.125 & 3.125 & 0.125 & $2,000 / 400$ \\
\hline 01-6-092 & 0.125 & 0.125 & 6.25 & 0.125 & $2,000 / 400$ \\
\hline Geometric mean & 0.111 & 0.151 & 4.961 & 0.140 & $1714.48 / 342.89$ \\
\hline
\end{tabular}

AMB: amphotericin B; FLC: fluconazole; ITR: itraconazole; VRZ: voriconazole. 
The MIC (geometric means) was $0.111 \mu \mathrm{g} / \mathrm{mL}$, $0.155 \mu \mathrm{g} / \mathrm{mL}, 4.819 \mu \mathrm{g} / \mathrm{mL}$ and $0.142 \mu \mathrm{g} / \mathrm{mL}$ for AMB, ITR, FLC and VRZ, respectively. The antimicrobial combination SMX/TMP was also able to inhibit the growth of all C. posadasii strains in vitro and the MIC values ranged from 1,000/200-2,000/400 $\mu \mathrm{g} / \mathrm{mL}$ (Table I). When used in combination with SMX/TMP, the MICs of the antifungals were reduced by 11, 2.4, 4.3 and 3.5 times for AMB, ITR, FLC and VRZ, respectively $(\mathrm{p}<0.05)$ (Table II). Sulpha derivatives are antimetabolic drugs that impair folic acid synthesis in microorganisms. These compounds have a broad antimicrobial spectrum and are active against several aerobic bacteria and some protozoan species and these compounds are frequently used successfully for the treatment and prevention of pneumonia caused by Pneumocystis jiroveci in acquired immune deficiency syndrome patients (Thomas et al. 2009). Although these compounds are not common first-line drugs in the management of fungal infections, the combination of SMX/ TMP has shown good results in treating paracoccidioidomycosis (Hahn et al. 2003, Wanke \& Aidê 2009). Our results show that SMX/TMP was able to enhance the susceptibility of $C$. posadasii to AMB by reducing the AMB MIC by approximately 5.85 times. Surprisingly, it was shown that the combination SMX/TMP alone was also able to impair $C$. posadasii growth in vitro.

In a previous work, Hanafy et al. (2007) found a great variability among SMX MICs against Cryptococcus sp. The authors reported that higher MIC values were attained when susceptibility tests were performed in organic-rich media because para-aminobenzoic acid (PABA) or PABA-related compounds may be present. In this study, we tried to perform the susceptibility tests with yeast nitrogen base, as suggested by Hanafy et al. (2007), but unfortunately this medium did not support the growth of $C$. posadasii (data not shown). Because other studies have already been performed with RPMI (Yekutiel et al. 2004, Navarro-Martinez et al. 2006), we decided to evaluate this medium in our experiments. However, we assumed that in PABA-free medium, SMX/ TMP may cause a greater reduction in AMB MICs.

We hypothesise that low concentrations of AMB may have caused slight damage to the fungal plasmatic membrane, allowing moderate ion leakage. In addition, these channels across the membrane may have allowed SMX/TMP to enter the cell. Therefore, we believe that acid folic blockade may be a potential target for C. posadasii inhibition. The design of sulpha drugs with higher affinity for dihydrofolate reductase or other folic acid biosynthetic pathway enzymes could enhance fungal in-

TABLE II

Minimum inhibitory concentration (MIC) of sulfamethoxazole plus trimethoprim (SMX/TMP) associated with antifungals against clinical and environmental Coccidioides posadasii strains

\begin{tabular}{|c|c|c|c|c|c|c|c|c|}
\hline \multirow{3}{*}{$\frac{\text { Strains }}{01-6-085}$} & \multicolumn{8}{|c|}{$\mathrm{MIC}(\mu \mathrm{g} / \mathrm{mL})$} \\
\hline & \multicolumn{2}{|c|}{ SMX/TMP + AMB } & \multicolumn{2}{|c|}{$\mathrm{SMX} / \mathrm{TMP}+\mathrm{ITR}$} & \multicolumn{2}{|c|}{$\mathrm{SMX} / \mathrm{TMP}+\mathrm{FLC}$} & \multicolumn{2}{|c|}{$\mathrm{SMX} / \mathrm{TMP}+\mathrm{VRZ}$} \\
\hline & $250 / 50$ & 0.007 & $250 / 50$ & 0.062 & $250 / 50$ & 0.781 & $500 / 100$ & 0.062 \\
\hline $01-6-087$ & $500 / 100$ & 0.015 & $500 / 100$ & 0.125 & $250 / 50$ & 0.781 & $250 / 50$ & 0.031 \\
\hline 01-6-088 & $500 / 100$ & 0.015 & $500 / 100$ & 0.125 & $500 / 100$ & 1.562 & $250 / 50$ & 0.031 \\
\hline 01-6-089 & $500 / 100$ & 0.015 & $250 / 50$ & 0.062 & $250 / 50$ & 0.781 & $250 / 50$ & 0.031 \\
\hline 01-6-090 & $500 / 100$ & 0.015 & $500 / 100$ & 0.125 & $500 / 100$ & 1.562 & $500 / 100$ & 0.062 \\
\hline 01-6-091 & $500 / 100$ & 0.015 & $250 / 50$ & 0.062 & $500 / 100$ & 1.562 & $500 / 100$ & 0.062 \\
\hline 01-6-092 & $500 / 100$ & 0.015 & $250 / 50$ & 0.062 & $250 / 50$ & 0.781 & $250 / 50$ & 0.031 \\
\hline 01-6-101 & $500 / 100$ & 0.015 & $500 / 100$ & 0.125 & $500 / 100$ & 1.562 & $500 / 100$ & 0.062 \\
\hline $01-6-102$ & $1,000 / 200$ & 0.031 & $250 / 50$ & 0.062 & $500 / 100$ & 1.562 & $500 / 100$ & 0.062 \\
\hline $01-6-103$ & $500 / 100$ & 0.015 & $250 / 50$ & 0.062 & $500 / 100$ & 1.562 & $250 / 50$ & 0.031 \\
\hline $05-02-063$ & $500 / 100$ & 0.015 & $250 / 50$ & 0.062 & $250 / 50$ & 0.781 & $250 / 50$ & 0.031 \\
\hline $05-02-064$ & $500 / 100$ & 0.015 & $250 / 50$ & 0.062 & $250 / 50$ & 0.781 & $250 / 50$ & 0.031 \\
\hline $05-02-065$ & $1,000 / 200$ & 0.031 & $500 / 100$ & 0.125 & $250 / 50$ & 0.781 & $500 / 100$ & 0.062 \\
\hline $05-02-066$ & $500 / 100$ & 0.015 & $250 / 50$ & 0.062 & $500 / 100$ & 1.5625 & $250 / 50$ & 0.031 \\
\hline $05-02-067$ & $500 / 100$ & 0.015 & $500 / 100$ & 0.125 & $500 / 100$ & 1.562 & $500 / 100$ & 0.062 \\
\hline $05-02-068$ & $500 / 100$ & 0.015 & $250 / 50$ & 0.062 & $250 / 50$ & 0.781 & $250 / 50$ & 0.031 \\
\hline $05-02-069$ & $500 / 100$ & 0.015 & $250 / 50$ & 0.062 & $250 / 50$ & 0.781 & $250 / 50$ & 0.031 \\
\hline $05-02-070$ & $1,000 / 200$ & 0.031 & $250 / 50$ & 0.062 & $500 / 100$ & 1.562 & $500 / 100$ & 0.062 \\
\hline
\end{tabular}

AMB: amphotericin B; FLC: fluconazole; ITR: itraconazole; VRZ: voriconazole. 
hibition. In fact, this pathway seems to be determinant of fungal viability, as Aspergillus fumigatus mutants defective in folate biosynthesis have been shown to be avirulent (Brown et al. 2000).

According to Navarro-Martinez et al. (2006), interruption of the folic acid biosynthetic pathway also impairs ergosterol production in Candida albicans, possibly by disturbing the metabolism of sterol $\mathrm{C} 24$ methyltransferase because the synthesis of its substrate is also blocked. It is well-known that ergosterol biosynthesis is also interrupted by azole antifungals agents that act on sterol $14 \alpha$-demethylase enzyme. The synergistic effect of these drugs was demonstrated in this study.

Although it is reasonable to suppose that the results of susceptibility tests against the parasitic form of $C$. posadasii are more reliable, very few studies have tested the susceptibility pattern of the yeast-like form of the fungus because the transition from mycelia to yeast it is not easily achieved (Hector et al. 1988). In contrast, tests with cells in the saprophytic phase are easier to perform and, even though CLSI protocols are not intended for testing dimorphic fungi, these protocols can be applied, generating results that are easy to compare.

The results obtained in this study demonstrate the potential of the SMX/TMP/AMB combination, which has an inhibitory effect on C. posadasii strains, even at sub-MIC concentrations of AMB. In addition, the results showed that SMX/TMP also has an inhibitory effect on C. posadasii, suggesting that acid folic blockade may be a potential antifungal target for C. posadasii. As far as we know, this is the first report of the antifungal potential of sulpha drugs against this pathogen.

\section{REFERENCES}

Afeltra J, Verweij PE 2003. Antifungal activity of nonantifungal drugs. Eur J Clin Microbiol Infect Dis 22: 397-407.

Brown JS, Aufauvre-Brown A, Brown J, Jennings JM, Arst H Jr, Holden DW 2000. Signature-tagged and directed mutagenesis identify PABA synthetase as essential for Aspergillus fumigatus pathogenicity. Mol Microbiol 36: 1371-1380.

Chapman SW, Sullivan DC, Clearly JD 2008. In search of the holy grail of antifungal therapy. Trans Am Clin Climatol Assoc 119: 197-115.

CLSI - Clinical and Laboratory Standards Institute 2008. Reference method for broth dilution antifungal susceptibility testing of filamentous fungi: approved standard, 2nd ed., document M38-A2. Available from: clsi.org/source/orders/free/M38-a2.pdf.

Cordeiro RA, Brilhante RS, Rocha MF, Bandeira SP, Fechine MA, de Camargo ZP, Sidrim JJ 2010. Twelve years of coccidioidomycosis in Ceará state, Northeast Brazil: epidemiologic and diagnostic aspects. Diagn Microbiol Infect Dis 66: 65-72.
Cordeiro RA, Brilhante RS, Rocha MF, Medrano DJ, Monteiro AJ, Tavares JL, de Lima RA, de Camargo ZP, Sidrim JJ 2009. In vitro synergistic effects of antituberculous drugs plus antifungals against Coccidioides posadasii. Int J Antimicrob Agents 34: 278-280.

Galgiani JN, Ampel NM, Blair JE, Catanzaro A, Johnson RH, Stevens DA, Williams PL, Infectious Diseases Society of America 2005. Coccidiodomycosis. Clin Infect Dis 41: 1217-1223.

González GM, González G, Najvar LK, Graybill JR 2007. Therapeutic efficacy of caspofungin alone and in combination with amphotericin B deoxycholate for coccidioidomycosis in a mouse model. J Antimicrob Chemother 60: 1341-1346.

Hahn RC, Morato CYT, Santos NL, Ferreira JF, Hamdan JS 2003. Disseminated paracoccidioidomycosis: correlation between clinical and in vitro resistance to ketoconazole and trimethoprim sulphamethoxazole. Mycoses 46: 324-329.

Hanafy A, Uno J, Mitani H, Kang Y, Mikami Y 2007. In vitro antifungal activities of sulfa drugs against clinical isolates of Aspergillus and Cryptococcus species. Jpn J Med Mycol 48: 47-50.

Hector RF, Zimmer BL, Pappagianis D 1988. Microtiter method for MIC testing with spherule-endospore-phase Coccidioides immitis. J Clin Microbiol 26: 2667-2668.

Johnson MD, Perfect JR 2010. Use of antifungal combination therapy: agents, order and timing. Curr Fungal Infect Rep 4: 87-95.

Kriesel JD, Sutton DS, Schulman S, Fothergill AW, Rinaldi MG 2008. Persistent pulmonary infection with an azole-resistant Coccidioides species. Med Mycol 46: 607-610.

Matsuda T, Koreeda Y, Mataki H, Taira T, Noma S, Higashimoto I 2010. A case of Aspergillus empyema successfully treated with combination therapy of voriconazole and micafungin: excellent penetration of voriconazole and micafungin into pleural fluid. Intern Med 49: 1163-1169.

Navarro-Martinez MD, Cabezas-Herrera J, Rodriguez-Lopez JN 2006. Antifolates as antimycotics? Connection between the folic acid cycle and the ergosterol biosynthesis pathway in Candida albicans. Int J Antimicrob Agents 28: 560-567.

Shubitz LF, Galgiani JN, Tian ZQ, Zhong Z, Timmermans P, Katz L 2006. Efficacy of ambruticin analogs in a murine model of coccidioidomycosis. Antimicrob Agents Chemother 50: 3467-3469.

Stevens DA, Rendon A, Gaona-Flores V, Catanzaro A, Anstead GM, Pedicone L, Graybill JR 2007. Posaconazole therapy for chronic refractory coccidioidomycosis. Chest 132: 952-958.

Thomas M, Rupali P, Woodhouse A, Ellis-Pegler R 2009. Good outcome with trimethoprim $10 \mathrm{mg} / \mathrm{kg} /$ day-sulfamethoxazole $50 \mathrm{mg}$ / $\mathrm{kg}$ /day for Pneumocystis jirovecii pneumonia in HIV infected patients. Scand J Infect Dis 41: 862-868.

Wanke B, Aidê MA 2009. Chapter 6 - Paracoccidioidomycosis. J Bras Pneumol 35: 1245-1249.

Yekutiel A, Shalit I, Shadkchan Y, Osherov N 2004. In vitro activity of caspofungin combined with sulfamethoxazole against clinical isolates of Aspergillus spp. Antimicrob Agents Chemother 48: 3279-3283. 